\title{
Accurate Mass Determination of Organotrifluoroborates
}

\author{
Daniel E. Petrillo, Rakesh K. Kohli, and Gary A. Molander \\ Roy and Diana Vagelos Laboratories, Department of Chemistry, University of Pennsylvania, Philadelphia, \\ Pennsylvania, USA
}

Exact mass measurements were obtained for a variety of potassium- and tetra- $n$-butylammonium organotrifluoroborates using commercially available organic sulfate salts as internal reference standards. Accuracies were determined within $5 \mathrm{ppm}$ using a sector ESI mass spectrometer operating in the negative ionization mode. (J Am Soc Mass Spectrom 2007, 18, 404-405) @ 2007 American Society for Mass Spectrometry

$\mathrm{P}$ olar, nonvolatile compounds of low molecular weight can be difficult to characterize by highresolution mass spectrometry (HRMS), although they often ionize well using electrospray mass spectrometry (ESIMS) [1]. Specifically, the determination of accurate mass below 300 amu is hindered because of the lack of commercially available reference standards when using sector instruments. Common reference standards such as cesium iodide [2,3] and sodium trifluoroacetate [4] provide suitable negative ions, but the peaks often are spaced too far apart for accurate mass determination when using a sector analyzer. Therefore a need exists for a set of readily available reference standards that can be applied to the characterization of polar molecules of low molecular weight.

As part of an ongoing research program in organoboron chemistry [5], we required HRMS data on several novel potassium- and tetra- $n$-butylammonium organotrifluoroborates $\left(\mathrm{RBF}_{3} \mathrm{~K}\right.$ and $\left.\mathrm{RBF}_{3}-\mathrm{NBu}_{4}\right)$ for complete characterization. Results from our group and others [6, 7] indicated that low-resolution LC-ESIMS operating in the negative ion mode allowed observation of the molecular anions of interest. However, this method does not provide the high degree of accuracy required for the determination of elemental composition. Although HRMS data have been obtained on similar compounds using fast atom bombardment (FAB) [8], such instrumentation was not readily available to us and thus we sought a method that could be implemented with the instrumentation on hand.

We reasoned that other low molecular weight compounds that easily form negative ions would act as suitable standards. Polyethylene glycol sulfates have been used in the past as internal standards for HRMS, albeit for

Published online November 16, 2006

Address reprint requests to Daniel E. Petrillo, University of Pennsylvania, Department of Chemistry, Box 177, 231 S. 34th St., Philadelphia, PA 19104-6323. E-mail: petrillo@sas.upenn.edu larger molecules [2]. Smaller sulfate salts such as sodium alkyl sulfates $\left(\mathrm{RSO}_{4} \mathrm{Na}\right)$ are readily available in the molecular weight range that we desired and differ by a methylene unit (14 Da), making it easy to bracket the molecular ion under examination. We chose to explore these compounds as potential internal reference standards.

\section{Experimental}

Mass spectra were obtained on a Micromass Autospec with a double-focusing magnetic sector analyzer operating in the negative electrospray ionization mode. Opus MassLynx version 3.5 software was used for analysis. Analytes and reference standards were introduced by infusion as solutions in methanol. Sodium ethyl sulfate and sodium n-propyl sulfate were purchased from City Chemical (West Haven, CT). All other standards were purchased from Alfa Aesar (Ward Hill, MA). Potassium- and tetra- $n$-butylammonium trifluoroborates were synthesized by standard methods [5].

\section{Results and Discussion}

Using commercially available sodium $n$-butyl sulfate and sodium $n$-hexyl sulfate as reference standards, we obtained an accurate mass of the anion of potassium $\beta$-styrenyltrifluoroborate (Table 1, entry 1) within an error range of $2.2 \mathrm{ppm}$. A variety of other potassium organotrifluoroborates were then examined using other commercially available sodium alkyl sulfates. In all cases, the error was $<5 \mathrm{ppm}$, making their characterization satisfactory for publication (Table 1). This is notable for the anion of potassium allyltrifluoroborate (Table 1, entry 2), which has a low mass of 109.0436. In this case the exact mass was determined within a $2 \mathrm{ppm}$ error range.

Other potassium trifluoroborates studied contained functionality such as an ester, epoxide, alcohol, aldehyde, and ketone. Alkyl-, alkenyl-, aryl-, and alkynyl trifluoroborates, which have been used in our research 
Table 1. Accurate masses of organotrifluoroborates

\begin{tabular}{|c|c|c|c|c|}
\hline Entry & Analyte & Calculated & Found & Error (ppm) \\
\hline 1 & & 171.0593 & 171.0589 & 2.2 \\
\hline 2 & & 109.0436 & 109.0435 & 1.2 \\
\hline 3 & & 151.0906 & 151.0905 & 0.5 \\
\hline 4 & & 187.0542 & 187.0535 & 3.9 \\
\hline 5 & & 173.0386 & 173.0384 & 0.8 \\
\hline 6 & & 139.0542 & 139.0547 & 3.4 \\
\hline 7 & & 169.1012 & 169.1007 & 2.5 \\
\hline 8 & & 189.0699 & 189.0697 & 0.7 \\
\hline 9 & & 273.1274 & 273.1274 & 0.1 \\
\hline 10 & & 211.0906 & 211.0912 & 3.1 \\
\hline
\end{tabular}

program [5], were all characterized by this technique. To date, we have used this technique to obtain HRMS data for publication on over 100 similar compounds in the course of our research.

\section{Conclusion}

Commercially available organic sulfates were used for the exact mass determination of potassium and tetra- $n$ butylammonium trifluoroborates. This method should find use for other polar, nonvolatile compounds of low molecular weight. It relies on standard equipment and uses widely available compounds as internal reference standards.

\section{Acknowledgments}

The authors thank the National Institute of General Medical Sciences (Grant GM-35249), Amgen, and Merck Research Laboratories for their generous support of this project. We also acknowledge Dr. Alex Young (University of Toronto) and John Dykins (University of Delaware) for helpful discussions.

\section{References}

1. Pramanik, B. N.; Ganguly, A. K., Gross, M. L., Eds.; Applied Electrospray Mass Spectrometry; Marcel Dekker: New York, 2002.

2. Cody, R. B.; Tamura, J.; Musselman, B. D. Electrospray Ionization Magnetic-Sector Mass Spectrometry-Calibration, Resolution, and Accurate Mass Measurements. Anal. Chem. 1992, 64, 1561-1570.

3. Hop, C. E. A. Generation of High Molecular Weight Cluster Ions by Electrospray Ionization: Implications for Mass Calibration. J. Mass Spectrom. 1996, 31, 1314-1316.

4. Moini, M.; Jones, B. L.; Rogers, R. M.; Jiang, L. Sodium Trifluoroacetate as a Tune/Calibration Compound for Positive-and Negative-Ion Electrospray Ionization Mass Spectrometry in the Mass Range of 100-4000 Da. J. Am. Soc. Mass Spectrom. 1998, 9, 977-980.

5. Molander, G. A.; Figueroa, R. Organotrifluoroborates: Expanding Organoboron Chemistry. Aldrichim. Acta 2005, 39, 49.

6. Clay, J. M.; Vedejs, E. Hydroboration with Pyridine Borane at Room Temperature. J. Am. Chem. Soc. 2005, 127, 5766-5767.

7. Yuen, A. K. L.; Hutton, C. A. Deprotection of Pinacolyl Boronate Esters via Hydrolysis of Intermediate Potassium Trifluoroborates. Tetrahedron Lett. 2005, 46, 7899-7903.

8. (a)Batey, R. A.; Thadani, A. N.; Smil, D. V. Potassium Alkenyl- and Aryltrifluoroborates: Stable and Efficient Agents for Rhodium-Catalyzed Addition to Aldehydes and Enones. Org. Lett. 1999, 1, 1683-1686. (b) Batey, R. A.; Quach, T. D. Synthesis and Cross-coupling Reactions of Tetra Alkylammonium Organotrifluoroborates. Tetrahedron Lett. 2001, 42, 9099-9103. (c) Thadani, A. N.; Batey, R. A. A Mild Protocol for Allylation and Highly Diastereoselective Syn or Anti Crotylation of Aldehydes in Biphasic and Aqueous Media Utilizing Potassium Allyl- and Crotyltrifluoroborates. Org. Lett. 2002, 4, 3827-3830. 